\title{
Primary carcinoid tumour of the testis: A case-report
}

\author{
Letterio D'Arrigo ${ }^{1}$, Angela Costa ${ }^{1}$, Filippo Fraggetta ${ }^{2}$, Antonio Cacciola ${ }^{1}$, Astrid Bonaccorsi ${ }^{1}$, \\ Francesco Savoca ${ }^{1}$, Francesco Aragona ${ }^{1}$ \\ ${ }^{1}$ Urology and ${ }^{2}$ Pathology Unit, Cannizzaro Hospital, Catania, Italy.
}

\begin{abstract}
Summary Testicular carcinoid tumours (TCT) account for less than $1 \%$ of all testicular neoplasms. A 17-year-old male underwent radical orchiectomy for a painful indurated and increased in size right testicle; a mixed echogenic mass, with a central homogeneous area surrounded by a hypoechoic edge with calcifications was found at ultreasound with increased vascularity at color Doppler examination. Biochemical markers were within normal limits. These symptoms are not specific and the majority of TCT are only diagnosed on histopathology.

Patients should undergo long-term biochemical and radiological follow-up given potential for delayed metastases, in one case 17 years after primary treatment.
\end{abstract}

KEY WORDS: Testicular neoplasms; Neuroendocrine tumour; Carcinoid.

Submitted 20 December 2013; Accepted 31 March 2014

\begin{abstract}
INTRODUCTION
Testicular carcinoid tumours (TCT) account for less than $1 \%$ of all testicular neoplasms (1). TCT may be a component of teratoma, or it may occur in pure form, either as a primary growth or as a metastasis from an extratesticular source.

Herein, we report a case of primary TCT presenting as a painful mass without features of carcinoid syndrome.
\end{abstract}

Case report is described in Supplementary materials posted on www.aiua.it

\section{Discussion}

The term "carcinoid" was introduced to distinguish a group of low malignant neoplasms of the small intestine arising from NE cells. Overall, 70 cases of TCT have been reported in literature, including primary forms, carcinoid differentiation within a mature teratoma and metastases from an extratesticular site.

The histogenesis of primary TCT is still debated as the presence of NE cells within the testis has not been described.

Several hypothesis have been proposed. Mai et al. (2) suggest that TCT originate from the same precursor cell from which Leydig cells derive; in Abbosh et al. opinion (3), the TCT results from a chromosomal abnormality. A scrotal mass, either painful or indolent, is the most common presenting symptom. A specific ultrasound (US) pattern is not reported: in most cases, an isoechoic mass with a peripheral hypoechoic rim (4) is found, in some cases scanty intralesional calcifications have been described (5).

In a literature review reported by Strooma and Delaere (6) (61 cases plus a personal one) 16\% of the patients had symptoms of carcinoid syndrome (diarrhoea, sweating, palpitations, bronchoconstriction, headache, flushing, abdominal pain) that occur when serotonin, produced by the tumour, is released into the systemic circulation (7).

Serotonin is metabolised to 5-HIAA and excreted through the urine; thus, it is advisable that any patient with vasoactive symptoms and a testicular mass should have a 24 hour urinary dosage of 5-HIAA prior surgery. However, these symptoms are not specific and the majority of TCT are only diagnosed on histopathology.

When a TCT is diagnosed, a metastasis or an extratesticular primary tumour should be excluded, especially if the TCT is larger than $4 \mathrm{~cm}$ and/or is associated to carcinoid syndrome. As most carcinoid tumours are found in the ileum and all six testicular carcinoid metastases mentioned by Strooma and Delaere (6) originated from the ileum, barium contrast studies, computed tomography (CT) scan and gastro-intestinal (GI) video-endoscopy can be used to search for primary intestinal tumours. Nowadays, I-111 labelled octreotide scintigraphy has replaced CT scan in localizing metastases with a sensitivity of up to $96 \%$ (8).

5-HIAA urinary levels must be measured in a 24 hour urine sample, although only $88 \%$ of all carcinoid tumours produce 5-HIAA (10).

Radical orchidectomy is the treatment of choice of TCT and is curative for testis-confined primary forms. A retroperitoneal lymphadenectomy is recommended when TCT is a component of teratoma. Adjuvant chemo/radiotherapy are known to have little effect on these tumours (9).

Patients should undergo long-term biochemical and radiological follow-up given potential for delayed metas- 
tases, in one case 17 years after primary treatment (5). Sutherland et al. (11) suggest three monthly 5-HIAA measurements for the first year after diagnosis and annually thereafter although disease progression may occurs in the absence of elevated urinary 5-HIAA levels. Serum chromogranin A, secreted by carcinoid tumours, correlates with relapse in GI carcinoids and may be of use in the follow-up of TCT (9).

Most primary TCT have good prognosis even if associated with mature teratoma.

A cancer-related death is reported only in patients with metastases from or to the testis.

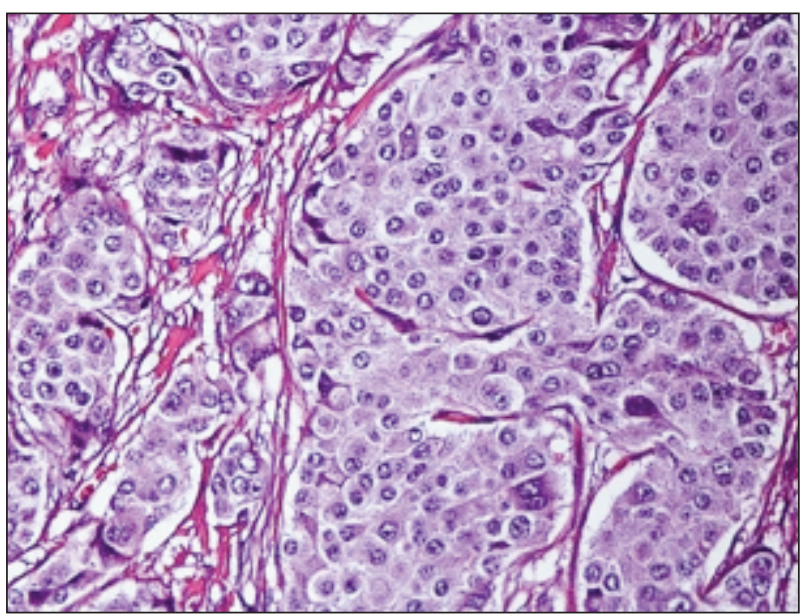

Figure 1.

The neoplastic cells were arranged in a nesting and trabecular pattern. No evidence of intratubular germ cell neoplasia. Tumor cells showed pale eosinophilic cytoplasm with round to oval nuclei and inconspicuous nucleoli; mitoses were not seen. No evidence of vascular invasion. The histological features were in accordance with a pure testicular carcinoid tumour without teratomatous components.

\section{REFERENCES}

1. Wang W, Guo C, Berney D, et al. Primary carcinoid tumour of thetestis: a clinicopathologic study of 29 cases. Am J Surg Pathol. 2010; 34:519.

2. Mai K, Park P, Yazdi H, Carlier M. Leydig cell origin of testicular carcinoid tumor: immunoistochemical and electron microscopic evicence. Histopathology. 2006; 49:548.

3. Abbosh P, Zhang S, MacLennan G, et al. Germ cell origin of testicular carcinoid tumors. Clin Cancer Res. 2008; 14:1393.

Figures and full list of References are posted in Supplementary materials on www.aiua.it

\section{Correspondence}

Letterio D'Arrigo, MD

ldarrigo@alice.it

Angela Costa, MD

ang.urolog@gmail.com

Antonio Cacciola, MD

tonycacciola@tiscali.it

Astrid Bonaccorsi, MD

astridbonaccorsi@libero.it

Francesco Savoca, MD

francescosavoca@virgilio.it

Francesco Aragona, MD

frank.aragona@virgilio.it

Urology Unit Cannizzaro Hospital Catania

Via Messina 829 - 95127 Catania, Italy

Filippo Fraggetta, MD

filippofra@hotmail.com

Pathology Unit Cannizzaro Hospital Catania

Via Messina 829 - 95127 Catania, Italy 